\title{
Disseminated Cutaneous Tuberculosis like Fungal Sporotrichosis Successfully Treated With Terbinafine: A Rare Case Report
}

\author{
Anil Gautam, ${ }^{1}$ Bijay Subedi, ${ }^{1}$ Janak Awasthi, ${ }^{2}$ Dr.Suman Adhikari ${ }^{3}$ \\ ${ }^{1}$ Faculty of Health Sciences, Pokhara University, Pokhara, Nepal; \\ ${ }^{2}$ Gandaki Medical College,Pokhara, Nepal; \\ ${ }^{3}$ National Academy of Medical Sciences, Kathmandu, Nepal. \\ Correspondence: \\ Anil Gautam, Email: gautamannil@gmail.com
}

Occurrence of Sporotrichosis is uncommon in Nepal. Here, we describe a case of cutaneous Sporotrichosis of 43 years old adult male working as a farmer from Pokhara-30, Nepal with unusual skin tuberculosis like presentation since 3 years, initiating from the unusual location in right pinna. Histopathological observation was suggestive of cutaneous tuberculosis, lupus vulgaris, cutaneous leishmaniasis due to overlapping findings during biopsy. The case was diagnosed by the pharmacological intervention observing the effect of drugs i.e., terbinafine. The purpose of reporting this serendipitous case is to enhance timely diagnosis, avoid the diagnostic dilemma for future references.

Keywords: Cutaneous tuberculosis; fungal; sporotrichosis; terbinafine.

Submitted: May 26, 2021 Accepted: June 22, 2021 Published: June 28, 2021

\section{INTRODUCTION}

Sporotrichosis is a rare form of fungal infection that is caused by the fungus Sporothrix schenkii due to accidental traumatic inoculation on the skin..$^{1,2}$ The fungus is worldwide in distribution and found in soil, organic decaying matter, barks of trees, and agricultural fields having the tropical and temperate climate. ${ }^{3}$ The transmission vulnerability is very high on people working in the farm, garden, mine, and immunocompromised person.1 The starting lesion is papule, pustule which finally ulcerate to form painless nodule. ${ }^{4}$ The high in-vivo activity of terbinafine suits itself as the mainstay for treatment of wide range of fungal infection. ${ }^{2}$

\section{CASE REPORT}

Here we describe a case of cutaneous sporotrichosis of 43 years old adult male after getting the informed consent, working on agricultural fields from Pokhara-30, Kaski District with a history of ear-piercing 12 years back. He presented the initial erythematous non-healing ulcer with no active bleeding and pus which progressed to granulomatous lesions of size 2-3 cm followed by multiple papulonodular lesions of different sizes and shape over the period of 6 months. The lesions appeared over the lymphatic channel on the right forearm and arm in a disseminated form. Unlike other cases which starts from the common sites such as hand, legs, knees, this peculiar case of sporotrichosis was first seen in the right pinna and later it spreads to the other sites of the body such as finger, forearm lesions. He was immunologically

Citation: Gautam A, Subedi B, Awasthi J, Adhikari S.Disseminated cutaneous tuberculosis like fungal sporotrichosis successfully treated with terbinafine: a rare case report. Nepal J Health Sci. 2021 Jan-Jun;1(1): 69-72 
competent and had no history of trauma at the lesion site. He had a history of ear piercing 12 years back for the purpose of gold ear ring, with no history of trauma. Initially, it was thought to be allergy of gold and was suggested to remove the gold ring from the ear and was prescribed skin ointment by $1^{\text {st }}$ Dermatologist. However, the lesions was not cured but it was much severe. According to the patient, he was then assigned to $2^{\text {nd }}$ Dermatologist who started empirical anti-tuberculosis therapy for 5 months despite having negative Mantoux test, cause the lesion was found to be mimicking cutaneous tuberculosis. There was no any progress of recovery even after 5 months of anti-tuberculosis therapy, however further deterioration of lesions and case was halted. The case was reviewed again with the help of $3^{\text {rd }}$ consultant Dermatologist and a differential diagnosis of Sporotrichosis, lupus vulgaris, and cutaneous leishmaniasis was made causing diagnostic dilemma. Biopsy was taken from the right forearm and sent to the histopathology laboratory. Along with that, the fungal culture and microscopy were done. The gross description of the specimen revealed the skin-covered tissue measuring $0.4 \mathrm{~cm}$ with the skin surfaces showing a tiny elevated area. Similarly, the histopathological finding revealed the tissue lined by stratified squamous epithelium with hyperkeratosis and pseudoepitheliomatous hyperplasia. Dermis showed the dense chronic inflammatory cells infiltrate comprising of lymphocytes, plasma cells, and few neutrophils. Occasional multinucleated giant cells along with scattered epithelioid cells and histiocytes were seen suggestive of cutaneous tuberculosis. However, the fungal culture after 7 days on $25^{\circ} \mathrm{C}$ and $37^{\circ} \mathrm{C}$ showed whitish colonies, moist and glabrous nature. Microscopy after Lactophenol Cotton Blue staining showed branched septate hyphae with conidia according to the laboratory report. Fungal structures of different morphological shapes were seen under Periodic acid Schiff (PAS) staining. Hence the differential diagnosis of Sporotrichosis was made by fungal culture, histopathological observation, macroscopic features of the lesion, and microscopy. After the differential diagnosis of Sporotrichosis, terbinafine 250 mg twice a day was prescribed for 6 months. The response was noted and observed periodically on the two month's basis. First two months of initiating the terbinafine therapy some 30\% recovery was evident. Similarly, the $4^{\text {th }}$ and $6^{\text {th }}$ months were characterized by $70 \%$ and $100 \%$ recovery leaving behind the permanent scar. The response of the terbinafine was rapid with the healing of the lesions leaving the scars at the end of the six months. However, the patient suffers the permanent distortion of shape and structure of the right pinna from the original structure.

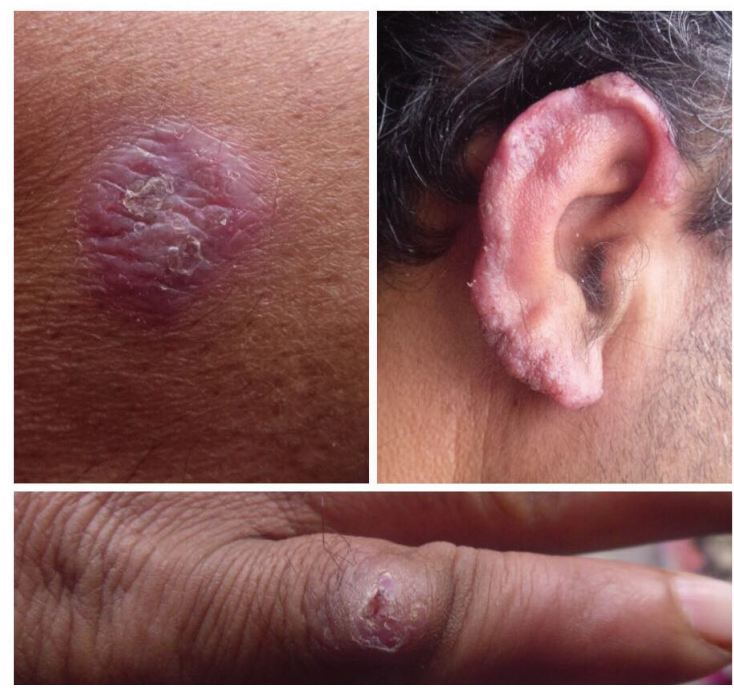

Figure 2: After the case was diagnosed and first two month of treatment with terbinafine 


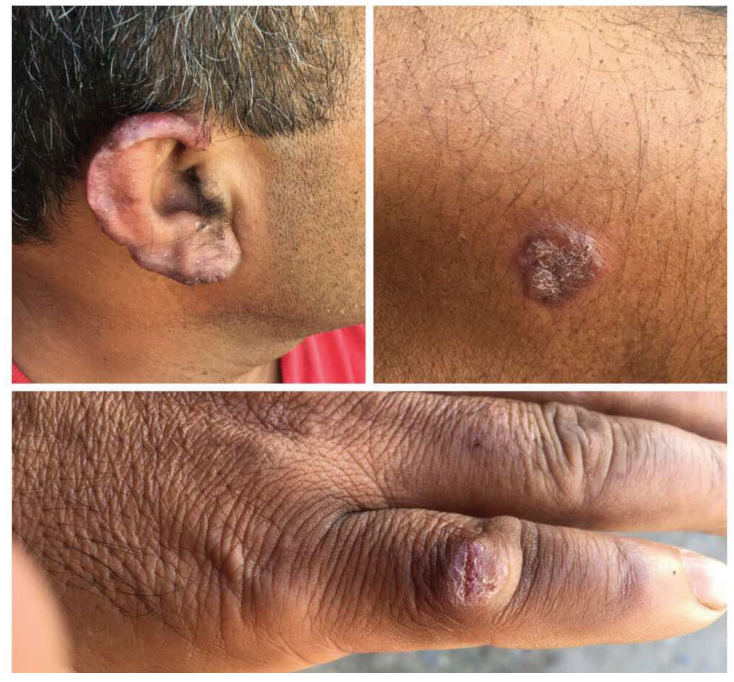

Figure 3: At the end of $4^{\text {th }}$ month of treatment
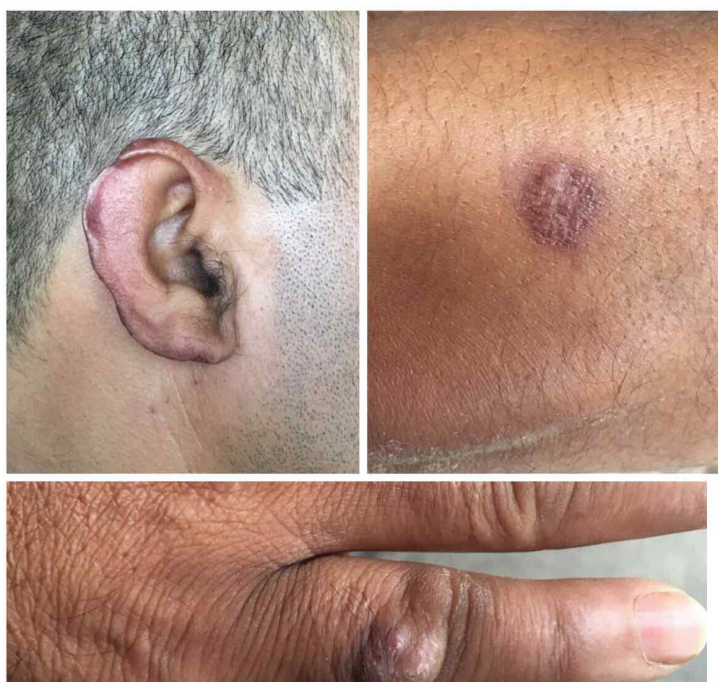

Figure 4: At the end of 6th month of successful treatment with terbinafine with the remnant of scars of the papule on the skin.

\section{DISCUSSION}

Sporotrichosis is a chronic form of fungal infection caused by Sporothrix schenckii in adults and rare in a child. ${ }^{5}$ The frequency of occurrence of sporotrichosis is common for people who are in close contact with contaminated agricultural products, soil, plants and living in tropical regions. ${ }^{6}$ Sporotrichosis is highly prevalent in the tropical and subtropical regions of the globe. ${ }^{2}$ Though sporotrichosis is uncommon in Nepal owing to its geographical location, tropical conditions and temperature the occupation of the patients with lack of safety gears, precautions favours sporotrichosis. Gyawali et al reported a first case of sporotrichosis in eastern Nepal. As per our literature review Reis BD et al has reported the sporotrichosis in usual location i.e., ear from Brazil with the history of ear piercing but it was not disseminating to all over the body unlike in our case report. According to the Fava Sc et al, among the four types of sporotrichosis cutaneous-lymphatic, fixed, disseminated and extra-cutaneous, disseminated cutaneous sporotrichosis is rare with occurrence only in $4 \%$ of patients with underlying immune suppression. However, in this case our patient was not immune compromised with no any history of chronic conditions. As we see from our case, Sporotrichosis shares its diverse overlapping symptoms and clinical features common with another case such as skin tuberculosis, cutaneous leishmaniasis, blastomycosis, it equally causes the diagnostic dilemma as well. ${ }^{2}$
Despite the wide availability of antifungal drugs, several authors including the Hull PR et al has reported the twice intake of terbinafine $250 \mathrm{mg}$ daily for 6 to 8 months as the best treatment option for cutaneous sporotrichosis for rapid cure. ${ }^{7}$ However, Keyong Guo et al and his team has combined the efficacy of itraconazole along with the terbinafine for fully treat the nasal sporotrichosis with continuous follow up of 8 months. So, the purpose of this case report with unusual position and unusual mode of the dissemination from right pinna of ear is to demonstrate the other form of sporotrichosis favouring the early diagnostic approach, treatment protocol and lessen the length of the disease. ${ }^{8}$

\section{SUMMARY}

According to the literature available to this date, this case report of sporotrichosis with atypical presentation is first of its kind reported in Western Nepal. The most vulnerable group i.e., agricultural farmers are at great risk of sporotrichosis due to lack of proper safety gears, awareness and campaign. Climate change and global warming are also favouring these types of tropical diseases shifting the occurrence of the diseases to new places. Untimely diagnosis of these types of serendipitous cases worsens the prognosis further.

\section{Conflict of interest : None}




\section{REFERENCE}

1. Motswaledi H, Nkosi L, Moloabi C, Nemutavhanan D, Maloba B. Sporotrichosis: A Case Report and Literature Review. J Clin Exp Dermatology. 2011;02(07):10-2. [ Full Text/ DOI ]

2. Coskun B, Saral Y, Akpolat N, Ataseven A, Demet C. Sporotrichosis successfully treated with terbinafine and potassium iodide : Case report and review of the literature. 2004;53-6. [PubMed | Full Text | DOI ]

3. Francis D, Freitas S, Hoagland BDES, Carlos A, Do F, Fraga BB. Sporotrichosis in HIV-infected patients : report of 21 cases of endemic sporotrichosis in Rio de Janeiro, Brazil. 2012;(February):170-8. [PubMed | Full Text ]

4. Davis BA. Sporotrichosis. Dermatol Clin 1996; 14: 69-76 [PubMed | DOI | Full Text ]

5. Neto Rda J, Machado AA, de Castro G, Quaglio AS, Martinez R. Disseminated cutaneous sporotrichosis as the initial manifestation of acquired immunodeficiency syndrome--case report. Rev Soc Bras Med Trop. 1999;32:57-61 [ PubMed ]

6. Tlougan BE, Podjasek JO, Patel SP, Nguyen XH, Hansen RC (2009) Neonatal sporotrichosis. Paediatr Dermatol 26: 563-565. [ PubMed | DOI | Full Text ]

7. Hull PR, Vismer HF. Treatment of cutaneous sporotri-chosis with terbinafine. Br J Dermatol 1992; 126: 51-55. [ PubMed | DOI | Full Text ]

8. Reis BD, Cobucci FO, Zacaron LH, D’Acri, AM, Lima RB, Martins CJ. Sporotrichosis in an unusual location: a case report. An Bras Dermatol. 2015;90 (3 Suppl 1): S84-7. [ PubMed/ Full Text/ DOI ] 